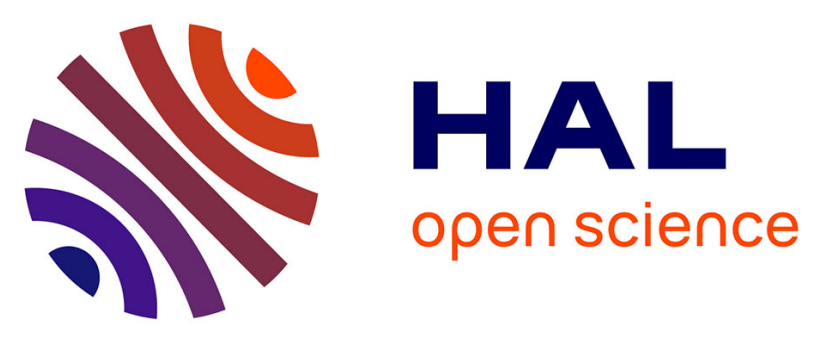

\title{
Etude de la viscosité de cisaillement des liquides sous-refroidis jusqu'à leur température de transition vitreuse. Analyse des variations thermiques des coefficients de Vogel-Fulcher. - I. Carbonate de propylène
}

\author{
Alberte Bondeau, J. Huck
}

\section{To cite this version:}

Alberte Bondeau, J. Huck. Etude de la viscosité de cisaillement des liquides sous-refroidis jusqu'à leur température de transition vitreuse. Analyse des variations thermiques des coefficients de Vogel-Fulcher. - I. Carbonate de propylène. Journal de Physique, 1985, 46 (10), pp.1717-1730. 10.1051/jphys:0198500460100171700 . jpa-00210124

HAL Id: jpa-00210124

https://hal.science/jpa-00210124

Submitted on 1 Jan 1985

HAL is a multi-disciplinary open access archive for the deposit and dissemination of scientific research documents, whether they are published or not. The documents may come from teaching and research institutions in France or abroad, or from public or private research centers.
L'archive ouverte pluridisciplinaire HAL, est destinée au dépôt et à la diffusion de documents scientifiques de niveau recherche, publiés ou non, émanant des établissements d'enseignement et de recherche français ou étrangers, des laboratoires publics ou privés. 


\title{
Etude de la viscosité de cisaillement des liquides sous-refroidis jusqu’à leur température de transition vitreuse. Analyse des variations thermiques des coefficients de Vogel-Fulcher. I. Carbonate de propylène
}

\author{
A. Bondeau \\ Laboratoire des Diélectriques Liquides. U.E.R. de Physique, \\ Université Lyon I, 43 bd du 11 Novembre 1918, 69622 Villeurbanne Cedex, France \\ et J. Huck \\ Laboratoire de Physique des Matériaux, U.E.R. de Sciences, \\ Université de Saint-Etienne, 23 rue du Docteur Paul Michelon, 42023 Saint Etienne Cedex, France
}

(Reçu le 28 mars 1985, accepté le 11 juin 1985)

\begin{abstract}
Résumé. - Nous avons mesuré la viscosité de cisaillement du carbonate de propylène dans l'intervalle $10^{-3}$ $10^{10} \mathrm{P} 1$, entre 343 et $157 \mathrm{~K}$, à l'aide de trois techniques : écoulement capillaire, rotation et pénétrométrie. L'analyse locale (au $2^{\mathrm{e}}$ ordre) des variations thermiques de la viscosité montre que les coefficients de Vogel-Fulcher forment un spectre continu. Ce résultat peut être interprété par les variations non linéaires du volume libre du modèle de percolation de Cohen et Grest.
\end{abstract}

\begin{abstract}
The shear viscosity of propylene carbonate has been measured in the interval $10^{-3}-10^{10} \mathrm{P} 1$, from 343 to $157 \mathrm{~K}$, by means of three methods : capillary, rotational and penetroviscometry. The local analysis (2nd order expansion) of the viscosity temperature dependence shows that the Vogel-Fulcher coefficients have a continuum spectrum. This result may be explained by non linear variations of the free-volume in the Cohen-Grest percolation model.
\end{abstract}

\section{Introduction.}

La relation (1) proposée il y a plus de 60 ans par Vogel [1], Fulcher [2] puis Tammann et Hesse [3] pour représenter les variations thermiques de la viscosité de cisaillement isobare des liquides et des verres usuels fondus, s'étant révélée très générale, de nombreux auteurs admettent actuellement qu'elle est susceptible de décrire aussi les variations de la plupart des coefficients de transport et de relaxation tels que la conductivité ionique, les divers temps de relaxation, diélectrique, mécanique, structurale, de spin-réseau en R.M.N., et le coefficient de diffusion moléculaire :

$$
\log X(T)=A_{x}+\frac{B}{T-T_{0}} \quad T>T_{0}>0
$$

$X$ représente une des grandeurs typiques des phénomènes de transport; $A_{x}, B$ et $T_{0}$ sont des constantes, $T$ est la température absolue.
La valeur critique $T_{0}$ est un pôle simple de $\log X$ et peut représenter soit un zéro de $X$, ce qui est le cas du coefficient de diffusion, de la fluidité, de la conductivité $(B<0)$ soit une singularité où $X$ diverge et devient infiniment grand (cas de la viscosité, du temps de relaxation; $B>0$ ).

La relation VFTH (1) n'est pas restée limitée au seul domaine des liquides [1, 3-23] et des verres fondus $[2,24-30]$ mais bien au contraire, elle n'a cessé de s'étendre progressivement aux milieux condensés les plus divers, et recouvre actuellement les polymères amorphes [31-3], les électrolytes [34], les verres métalliques [35-6] les cristaux liquides [37-40], vitreux [41], ferroélectriques [42]. Elle a aussi été utilisée par Angell [43] pour établir une classification des liquides fondée sur leurs propriétés thermodynamiques au voisinage de la transition vitreuse. Il semble ainsi tout à fait naturel d'attribuer à (1) un caractère universel en physique des milieux désordonnés et notamment dans le domaine des liquides surfondus [44] où $T_{0}$ 
est souvent considérée comme la limite théorique de la température de transition vitreuse $T_{\mathrm{g}}$ qui serait observée au cours de refroidissements de plus en plus lents du liquide en équilibre [20, 45]. L'accroissement des temps de relaxation rend d'autre part cette limite inaccessible en pratique.

La généralité de (1), qui d'ailleurs n'est pas reconnue par tous les auteurs ne saurait cependant masquer les nombreuses difficultés que soulève l'emploi de cette relation tant dans le domaine théorique que dans le domaine expérimental.

Les modèles classiques qui permettent de lui donner un fondement théorique sont ceux du volume libre de Cohen et Turnbull $[8,47]$ et des relaxations coopératives de Gibbs, Di Marzio [48] et Adam [49] : $T_{0}$ est alors la température de transition thermodynamique au-dessous de laquelle le volume libre, la fluidité et l'entropie de configuration s'annulent, ce qui donne ainsi une solution possible au paradoxe de Kauzmann [50]. Ces modèles ont suscité diverses critiques [37-8, 51-8]. L'existence même d'une transition du $2^{\mathrm{e}}$ ordre [48-9] a été mise en doute car elle semble ne résulter que d'un traitement approximatif d'un problème statistique complexe. Quant au modèle du volume libre, il souffre non seulement de l'ambiguité de ce concept (différentes définitions ont pu être utilisées) et de son incompatibilité avec l'équation de van der Waals, mais aussi de son inaptitude à rendre compte des variations de $T_{\mathrm{g}}$ avec la pression. On peut noter par ailleurs qu'il vient d'être considérablement modifié par Cohen et Grest [59-61] dont la nouvelle théorie utilise maintenant un volume libre tout à fait distinct du volume libre "classique " puisqu'il reste fini, ainsi que la fluidité, à toute température non nulle.

Les problèmes liés à l'interprétation de $T_{0}$ et à ses implications dans le domaine des verres et des verres de spin ont été exposés par Anderson [56] Joffrin [63] et Toulouse [64]. Diverses explications faisant intervenir l'amplitude des fluctuations d'énergie [65-6] ou l'existence de défauts topologiques ont été avancées. Selon Duffy et Rivier [67] la relation VFTH pourrait décrire l'évolution aux températures élevées de la concentration des disclinations qui sont à l'origine des systèmes à deux niveaux expliquant les propriétés thermiques, acoustiques et diélectriques des verres $[56,63]$. On peut noter que les questions relatives à l'existence et à la signification de $T_{0}$, importantes pour la compréhension de la transition vitreuse, demeurent encore actuellement sans réponse, et que la situation qui en découle est de ce fait considérée comme très ouverte.

Sur le plan expérimental la situation n'apparaît pas très claire non plus, car l'ajustement des données expérimentales ne semble réellement satisfaisant que pour un nombre limité de composés $[2,3,6,14-5,21$, 24-6, 28, 37-8], et ce dans des intervalles relativement peu étendus. Il a en effet été constaté depuis longtemps déjà, qu'un seul jeu de coefficients $A, B, T_{0}$ était en général très insuffisant pour obtenir un ajustement valable, et cela a conduit à effectuer des lissages par intervalles partiels en distinguant au moins deux domaines, à « haute » et « basse température ", voire trois $[9-12,17,59,60,62,68-73]$. A la suite des travaux de Laughlin et Uhlmann [17] on a généralement admis [60-2, 73] qu'à " basse température » le régime d'écoulement pouvait être du type d'Arrhenius $\left(T_{\text {oII }}=0\right)$ ou de Vogel $\left(T_{\text {oII }} \neq 0\right)$ tandis qu'à " haute température " $\left(\eta \lesssim 10^{4} \mathrm{Pl}\right)$, il suivait (1) avec une température caractéristique $T_{0 I} \neq 0$. D'autres auteurs considèrent que la relation VFTH n'est valable que dans le seul domaine des faibles viscosités, loin de la zone de transition vitreuse [46]. Ces considérations ne sont visiblement pas en accord avec les résultats obtenus dans les polymères amorphes, où, au voisinage de $T_{g}$, la viscosité obéit à la relation de Williams-Landel-Ferry [31] dont la formulation est strictement équivalente à (1). Bien qu'il soit maintenant établi que les valeurs de ses coefficients ne sont pas universelles $[45,49,74-77]$ la relation WLF permet néanmoins de localiser $T_{0}$, quelques dizaines de degrés en-dessous de $T_{\mathbf{g}}$, tandis que (1) peut conduire à des valeurs de $T_{\mathrm{OH}}$ proches de $T_{\mathrm{g}}$, voire supérieures, comme c'est le cas pour o-terphényle, tri- $\alpha$-naphtylbenzène, le salol et divers fluorozirconates [30]. Lorsqu'il en est ainsi $\left(T_{\mathrm{OH}} \gtrsim T_{\mathrm{g}}\right)$ l'identification de $T_{\mathrm{OH}}$ à la température de disparition de l'entropie excédentaire [22, 37] n'est alors pas fondée. On voit donc que la généralité de (1) masque en réalité de sérieuses divergences quant à la localisation de $T_{0}$ et sa corrélation avec $T_{g}$.

Enfin on peut aussi noter que (1) n'est pas la seule relation susceptible de décrire les variations de la viscosité ou d'autres coefficients de transport. Des dizaines de modèles, relations empiriques ou semiempiriques ont été proposés depuis longtemps [4] et continuent de l'être [58-60, 62, 78-85].

On dispose donc actuellement d'une grande diversité de modèles qui sont susceptibles de conduire à des interprétations très différentes, voire opposées de la nature thermodynamique de la transition vitreuse. Existe-t-il ou non une transition de phase, et si oui quel est son ordre ?

D’après Cohen et Grest [59-62] les résultats expérimentaux ne permettent pas encore de donner une réponse précise à cette question, mais le modèle qu'ils proposent prédit une transition de phase du 1 er ordre.

Il paraît donc légitime de se demander si la précision des mesures actuelles est suffisante pour faire un choix significatif entre les modèles actuels, ce qui contribuerait ainsi à clarifier la situation. C'est pour tenter de répondre à cette question que nous avons procédé à une analyse des données expérimentales disponibles concernant la viscosité, avec pour objectif initial l'étude de la compatibilité des résultats expérimentaux avec l'hypothèse d'un pôle simple de $\log \eta$. Cette analyse a porté sur des données d'origine 
bibliographique publiées durant les 60 dernières années, et sur des données originales obtenues à l'aide d'un appareillage que nous avons réalisé au laboratoire pour mesurer la viscosité des liquides sous-refroidis entre 350 et $77 \mathrm{~K}$, dans la gamme $10^{-3}$ à $10^{12} \mathrm{P} 1$. Nous avons étudié la viscosité de plusieurs dizaines de composés [86] : alcanes et dérivés halogénés, hydrocarbures et diesters aromatiques, phénols, polymères amorphes, verres (silicates, anhydride borique, fluorozirconates). Les mesures effectuées au laboratoire concernent le carbonate de propylène, le glycérol et les binaires eau-glycérol, diverses huiles paraffiniques et naphténiques, et sont poursuivies actuellement sur d'autres composés (diesters aromatiques et alcools). L'ensemble des valeurs traitées permet d'apprécier les variations de la viscosité sur une large échelle de viscosité (pouvant atteindre 15 décades) et de température (entre 1700 et $100 \mathrm{~K}$ ). Le résultat essentiel de cette analyse est que les coefficients de (1) n'apparaissent pas en général être des constantes intrinsèques du liquide, mais bien plutôt des fonctionnelles de l'intervalle de température sélectionné, et représentent ainsi selon l'amplitude de cet intervalle des valeurs moyennes ou quasi-locales de fonctions $A(T), B(T), T_{0}(T)$, dont on peut établir, lorsque les données sont suffisantes, une expression analytique approchée. La relation de Vogel-Fulcher apparaît alors être une relation d'interpolation locale seulement, qui très souvent (sauf peut-être pour les viscosités les plus faibles) est sensiblement équivalente à une approximation du $2^{\mathrm{e}}$ ordre de $\log \eta$. Ce résultat est discuté dans le cadre des modèles classiques de volume libre et du modèle de Cohen et Grest.

Dans cet article, nous exposons les principes de la méthode expérimentale et du traitement des données utilisées, et nous présentons les résultats concernant le carbonate de propylène, dont les variations de la viscosité nous paraissent typiques et représentatives d'une large classe de liquides.

\section{Dispositif expérimental.}

Le dispositif de mesure de la viscosité de cisaillement des liquides newtoniens sous-refroidis entre 350 et $77 \mathrm{~K}$ comprend trois appareils fonctionnant dans les plages suivantes : $10^{-3}-10^{-2} ; 10^{-2}-10^{2} ; 10^{3}-10^{12} \mathrm{Pl}$.

Entre $10^{-3}$ et $10^{-2} \mathrm{Pl}$ il s'agit d'un viscosimètre capillaire à niveau suspendu de type Ubbelhode [87] plongé dans un bain thermostaté (350 à $273 \mathrm{~K}$ ). Dans la gamme $10^{-2}-10^{2}$ Pl les mesures sont effectuées à l'aide d'un viscosimètre rotatif VTO2 de Haake, placé dans un cryostat à convection (azote gazeux).

Enfin, au-dessus de $10^{3} \mathrm{Pl}$ nous utilisons un appareillage réalisé au Laboratoire, dont la description a déjà été donnée $[86,88]$. Son principe est le suivant : la profondeur d'enfoncement $h(t)$ d'une aiguille cylindrique chargée, est mesurée par un capteur électromagnétique de déplacement, enregistrée sur mémoire magnétique et traitée par un microordinateur. La résolution de l'équation de Navier-Stokes en régime quasi permanent laminaire [89] permet de calculer la viscosité absolue de cisaillement d'un fluide newtonien incompressible :

$$
\eta=\frac{c}{b}
$$

où $b$ représente la pente de $h^{2}(t)$ et $c=F / K ; K$ est un facteur géométrique ne dépendant que du rapport $x(x>1)$ des rayons $R_{2}$ de la nacelle cylindrique contenant le liquide et $R_{1}$ de l'aiguille de pénétration, tandis que $F$ est la charge (constante) de l'aiguille :

$$
K=\frac{\pi\left(1+x^{2}\right)}{\left(1+x^{2}\right) \ln x+1-x^{2}}\left(1-\frac{1}{x^{2}}\right) .
$$

Le facteur $1-1 / x^{2}$ tient compte du volume du liquide déplacé par l'aiguille. Le schéma du dispositif est représenté figure 1 .

La nacelle contenant le liquide $\left(R_{2}=12 \mathrm{~mm}\right.$, hauteur $40 \mathrm{~mm}$ ) est placee dans un cryostat à convection fabriqué par la Société des Matériels Cryogéniques. La température de l'azote gazeux est réglée par un thermaliseur, et la régulation thermique de l'ensemble est assurée par un programmateur de température Arion de type proportionnel-intégraldifférentiel. Pour la mesure des températures on utilise des sondes à résistance de platine Claral (norme DIN 43760) alimentées par un générateur de courant d'intensité constante $(1 \mathrm{~mA})$. Le gradient thermique est inférieur à $0,1 \mathrm{~K} / \mathrm{cm}$. Les mesures de viscosité sont

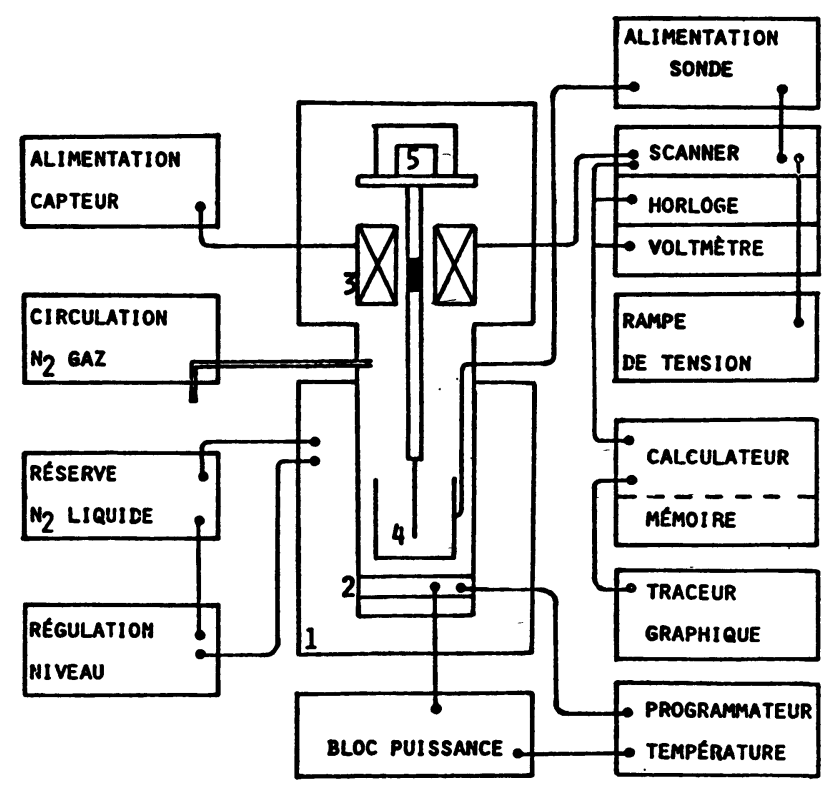

Fig. 1. - Dispositif de mesure de la viscosité par pénétrométrie [88], 1 : cryostat; 2 : thermaliseur; 3 : capteur de déplacement; 4 : cellule; 5 : masse de charge.

[General arrangement of the penetroviscometer, 1 : cryostat; 2 : heating apparatus; 3 : displacement captor; 4 : cell; 5 : load mass.] 
entreprises lorsque le régime isotherme est atteint, et que la température est stabilisée à $0,1 \mathrm{~K}$. Leur durée peut s'étendre entre quelques secondes et plusieurs dizaines d'heures, au cours desquelles la dérive n'a jamais excédé $0,3 \mathrm{~K}$. L'aiguille de pénétration $\left(R_{1}=0,5\right.$ à $3 \mathrm{~mm}$, hauteur $\left.30 \mathrm{~mm}\right)$ est fixée à un équipage mobile d'axe vertical (masse $37 \mathrm{~g}$ à $4 \mathrm{~kg}$ ), son déplacement est mesuré à l'aide de capteurs Schaevitz (HR-DC). Il s'agit de transformateurs différentiels à variations linéaires constitués d'un noyau ferromagnétique cylindrique solidaire de l'équipage mobile, qui canalise le flux magnétique entre des bobinages fixes. Le signal induit par le capteur est proportionnel au déplacement du noyau. La résolution $(10 \mu \mathrm{V})$ du voltmètre digital prélevant le signal (Datron 1041) permet de détecter un déplacement de 3,7 $\mu \mathrm{m}$. Les signaux délivrés par le capteur de déplacement et la sonde de température sont prélevés par un voltmètre associé à un scanner (Datron 1200) à des intervalles de temps $\Delta t$ réglables de $178 \mathrm{~ms}$ à $1,8 \times 10^{3} \mathrm{~s}$ et les valeurs correspondantes $h(t)$, $T(t), t$ sont stockées sur cassettes magnétiques et traitées par un microordinateur (HP 9815). On dispose aussi des enregistrements graphiques de $h(t)$, $h^{2}(t)$ et $T(t)$, ce qui permet de contrôler l'évolution thermique de la cellule et la linéarité de $h^{2}(t)$, et d'éliminer les régions affectées par le régime transitoire et les effets de bord (proximité du fond). Tant que la viscosité est inférieure à environ $10^{8} \mathrm{Pl}$, le graphique $h^{2}(t)$ est parfaitement linéaire depuis l'origine $h=0$, jusqu'à quelques millimètres du fond, zone où les tubes de courant ne sont plus cylindriques. Pour des viscosités plus élevées, il apparaît une $2^{\mathrm{e}}$ région non linéaire au début de l'enfoncement, en relation avec un régime transitoire de plus en plus long qui dépend des propriétés viscoélastiques du produit. En dehors de ces zones perturbées où (2) n'est pas valable, la linéarité de $h^{2}(t)$ est excellente comme l'indique la valeur du coefficient de corrélation égale à 1 , à $10^{-4}$ ou $10^{-5}$ près.

L'appareil a été testé avec du phényle salicylate, et les valeurs absolues de $\eta$ calculées d'après (2) ont été comparées à celles obtenues par Laughlin et Uhlmann [17] en utilisant la technique de flexion de barre. Les deux courbes représentatives de $\log \eta$ sont pratiquement confondues entre $10^{3}$ et $10^{8} \mathrm{Pl}$. Au-delà, bien que les valeurs de $\eta$ diffèrent (leur rapport peut atteindre 2,5 aux environs de $10^{12} \mathrm{Pl}$ ) la concordance est encore très acceptable puisque les températures de transition vitreuse $\left(\eta=10^{12} \mathrm{Pl}\right)$ ne diffèrent que de $1,5 \mathrm{~K}$ et que celles correspondant à des valeurs normalisées de $\eta\left(10^{4}, 10^{6}, 10^{8}, 10^{10} \mathrm{Pl}\right)$ présentent des écarts de l'ordre de quelques dixièmes de degrés ou moins seulement.

\section{Traitement des données.}

Lorsqu'on analyse les variations de la viscosité sur une quinzaine de décades, entre $10^{-3}$ et $10^{12} \mathrm{Pl}$, on constate aisément qu'à cette échelle il n'y a pra- tiquement pas de composés dont l'écoulement suive la relation d'Arrhenius. Les seuls composés paraissant avoir un comportement qui en soit assez proche sont ceux formant des réseaux covalents tels que $\mathrm{SiO}_{2}$ et $\mathrm{GeO}_{2}$ [90-92]; encore convient-il de noter que ces cas restent rares et peu probants à cause d'une répartition très inégale des mesures, et de l'influence des impuretés qui perturbent fortement les valeurs de la viscosité [92]. Au contraire, un comportement arrhénien est toujours trouvé lorsque les mesures sont effectuées sur des intervalles de faible amplitude. Il est donc naturel de considérer qu'un tel régime ne constitue en fait qu'une approximation locale, correspondant à un développement suivant $T^{-1}$, limité au $1^{\text {er }}$ ordre, de la fonction inconnue $\varphi=\log \eta$ dont on cherche à établir une expression analytique approchée compatible avec la précision expérimentale. Il est alors commode de poser $T^{*}=10^{3} T^{-1},\left(T^{*}\right.$ varie entre 0,6 et 10 environ) :

$$
\log \eta=A_{0}+B_{0} T^{*} \quad\left(1^{\mathrm{er}} \text { ordre }\right)
$$

On pourrait tenter d'exprimer $\varphi\left(T^{*}\right)$ à l'aide d'ajustements locaux du $1^{\mathrm{er}}$ ordre, mais la précision est alors très médiocre, car la faible amplitude des intervalles d'étude $\Delta T^{*}$ implique des ajustements effectués avec un nombre de données en général trop faible pour qu'ils soient significatifs. L'ajustement de la viscosité suivant des droites d'Arrhenius a d'autre part été utilisé depuis longtemps mais pour définir des températures de transition liquide-liquide $T_{11}$. L'inexistence de telles transitions vient d'être démontrée récemment par Orbon [93] dans le polystyrène. A grande échelle, il est manifeste que les points anguleux introduits par ce procédé de linéarisation apparaissent surtout comme des artifices d'ajustement.

L'approche que nous proposons consiste à ajuster localement les données expérimentales suivant la relation parabolique $(\mathrm{P})$ :

$$
\log \eta=a+b T^{*}+c T^{* 2} \quad\left(2^{\mathrm{e}} \text { ordre }\right) .
$$

Ce faisant on obtient le développement au $2^{\mathrm{e}}$ ordre de $\varphi\left(T^{*}\right)$ indépendamment de tout modèle théorique. Pour déterminer chaque intervalle partiel où ce développement est valable, on procède à l'examen de l'écart type $\delta p$ de l'ajustement et à l'analyse du caractère aléatoire ou non de la distribution des rapports des valeurs expérimentales et ajustées de la viscosité $\eta_{i} / \eta$.

La relation de Vogel-Fulcher est alors traitée comme une relation d'ajustement hyperbolique $(\mathrm{H})$ dont on peut définir le domaine de validité par comparaison systématique avec (5).

Nous avons élaboré un programme de traitement qui donne dans tout intervalle partiel sélectionné $\left(T_{i}^{*}, T_{j}^{*}\right)$ :

- les paramètres d'ajustement $a, b, c, A, B, T_{0}-$ les écarts-types et les résidus des estimateurs $(\mathrm{P})$ et $(\mathrm{H})$ 
- les paramètres statistiques des résidus

- les énergies locales d'activation - les températures correspondant aux valeurs normalisées classiques de la viscosité [45] - le tracé automatique des courbes $(\mathrm{P})$ et $(\mathrm{H})$ et de l'énergie d'activation - le diagramme de $\delta_{\mathrm{H}}\left(T_{0}\right)$ et des rapports $\mathrm{e}_{i}=\eta_{i} / \eta$.

L'ajustement (P) est effectué suivant une régression polynomiale classique à trois paramètres traitée par la méthode des moindres carrés, et l'ajustement hyperbolique $(\mathrm{H})$ selon une régression linéaire à 2 paramètres, en choisissant $T_{0}$ comme variable auxiliaire. Pour chaque composé, l'intervalle complet de température $\left(T_{1}^{*}, T_{2}^{*}\right)$ est systématiquement découpé en deux types d'intervalles partiels, d'origine variable et d'amplitude sensiblement constante, et d'origine fixe et d'amplitude variable $\left(T_{1}{ }^{*} \leftarrow\right)$ et $\left(\rightarrow T_{2}^{*}\right)$.

Ce double balayage (Fig. 2) permet ainsi d'observer la stabilité éventuelle des paramètres d'ajustement, et dans le cas contraire d'étudier leur évolution en fonction de la température. La figure 3 montre le diagramme obtenu en traitant les valeurs de la viscosité calculées à l'aide de (1) avec les valeurs suivantes des paramètres : $A=-8, B=10^{3}, T_{0}=100$. L'aspect typique des diagrammes, invariance de $T_{0}$, nullité de $\delta_{\mathrm{H}}$, variation de $\delta_{\mathrm{p}}$, caractérise un composé dont la viscosité, mesurée sans aucune erreur, obéirait à la relation (1) dans toute la gamme $10^{-3}-10^{12} \mathrm{Pl}$. La prise en compte de la précision des mesures expérimentales se manifeste simplement par des fluctuations aléatoires de $T_{0}(T)$, de part et d'autre de sa valeur moyenne, et par une valeur finie de l'écart type $\left(\delta_{\mathrm{H}} \neq 0\right)$ caractérisant l'erreur de la mesure. La figure 4 représente les variations de $\delta_{\mathrm{H}}\left(T_{0}\right)$ en fonction de la variable auxiliaire $T_{0}$, et leur évolution selon les fenêtres d'observation utilisées au cours des balayages. On constate que l'acuité du minimum disparaît progressivement lorsque l'amplitude de l'intervalle $\Delta T$ décroît. Pour une fenêtre trop étroite, la courbe $\delta_{\mathrm{H}}\left(T_{0}\right)$ présente non plus un minimum

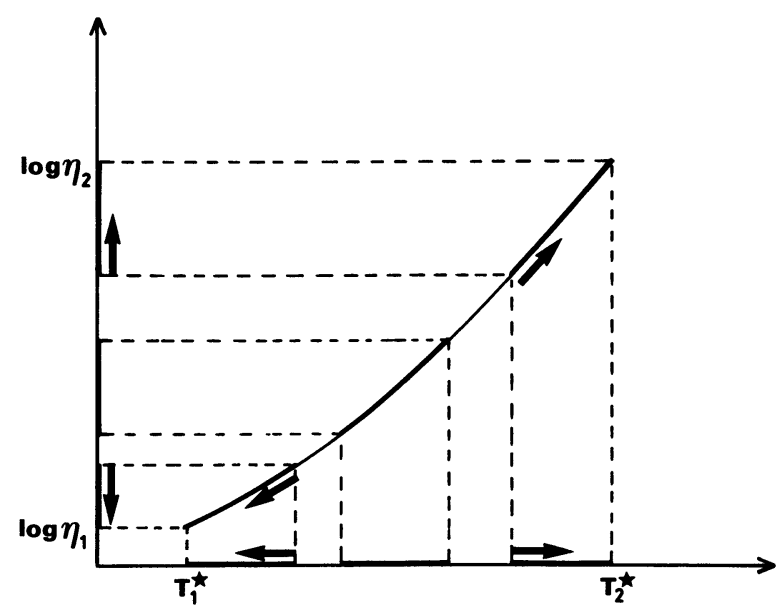

Fig. 2. - Mode de découpage en intervalles partiels.

[Partitioning into partial intervals.]

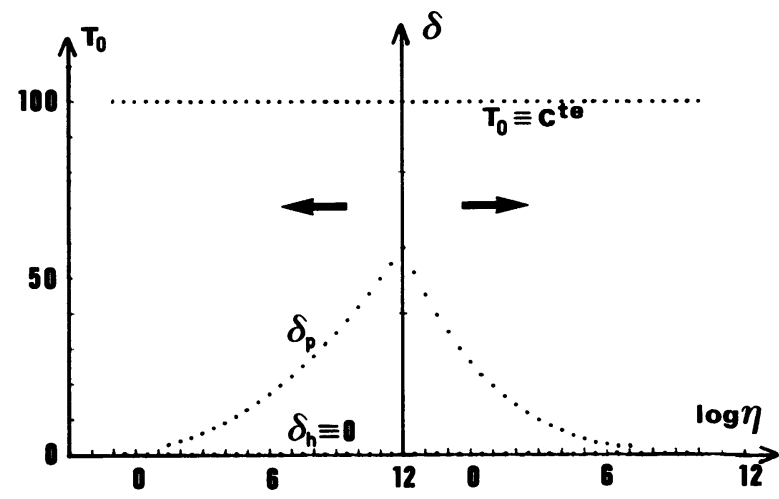

Fig. 3. - Traitement des données calculées d'après (1) avec les valeurs numériques : $A=-8, B=10^{3} ; T_{0}=100$.

[Fitting of the data calculated from (1) with the numerical values : $A=-8 ; B=10^{3} ; T_{0}=100$. $]$
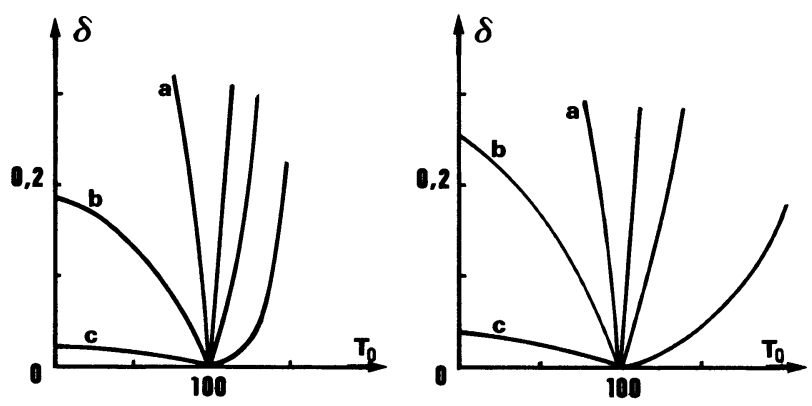

Fig. 4. - Variation de $\delta_{\mathrm{H}}\left(T_{0}\right)$ avec l'amplitude de l'intervalle de température.

(1) $T_{2}=150 \quad T_{i}=300(\mathrm{a}) ; \quad 180$ (b) 155 (c)

(2) $T_{1}=300 T_{i}=150(\mathrm{a}) ; \quad 183$ (b) 225 (c)

[Dependence of $\delta_{\mathrm{H}}\left(T_{0}\right)$ to the temperature interval width.]

bien localisé, mais un méplat pouvant s'étendre sur quelques dizaines voire quelques centaines de degrés. Dans ce cas la détermination de $T_{0}$ perd toute signification puisqu'il est possible d'effectuer des ajustements sensiblement équivalents avec des jeux de paramètres très différents. Cela est illustré figure 5 . La localisation de $T_{0}$ est bonne et la détermination des paramètres unique pour le diméthylphtalate [9]. Avec un verre de silicate, NBS 710 [25] toutes les valeurs de $T_{0}$ comprises entre 400 et 600 sont également admissibles et conduisent à des ajustements de même précision $\left(\delta_{\mathrm{H}} \simeq 4,5 \times 10^{-2}\right)$.

L'examen de $\delta_{\mathrm{H}}\left(T_{0}\right)$ permet de ne retenir que les ajustements dont la détermination des coefficients est unique. En pratique $T_{0}$, calculé à $1 \mathrm{~K}$ près, est localisé dans un intervalle $\Delta T_{0}$ n'excédant pas quelques degrés. L'analyse statistique des résidus, la comparaison des écarts types $\delta_{\mathrm{P}}$ et $\delta_{\mathrm{H}}$ permettent alors d'estimer si les ajustements sont compatibles ou non avec la précision expérimentale. 


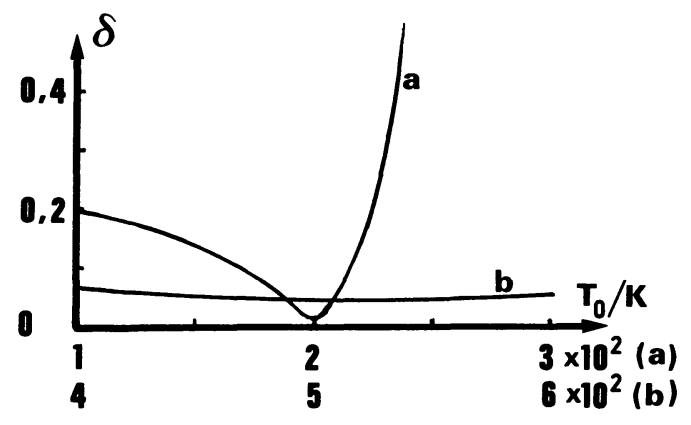

Fig. 5. - Variations de $\delta_{\mathrm{H}}\left(T_{0}\right)$ : (a) dimethylphtalate : 370-224 K [9], (b) verre NBS 710 : 973-831 K [25].

[Variation of $\delta_{\mathrm{H}}\left(T_{0}\right):$ (a) dimethylphtalate, (b) NBS 710 glass.]

\section{Viscosité du carbonate de propylène.}

Une revue des principales propriétés physicochimiques du carbonate de propylène $(\mathrm{CP})$ étudié à des températures supérieures à celle de sa fusion $\left(T_{\mathrm{f}} \simeq 223 \mathrm{~K}\right)$ a été donnée par Jasinski [94]. La molécule de CP possède un moment dipolaire élevé $\mu=5 D$ [95] donnant au liquide, à température ambiante, une permittivité voisine de $65[94,96]$<smiles>CC1COC(=O)O1</smiles>

Il n'existe pas d'associations moléculaires comme le montrent des mesures en I.R. et R.M.N. [97] et la valeur toujours proche de 1 du coefficient de corrélation de Kirkwood [96-100]. CP peut être surfondu, sans subir de trempe, avec des vitesses de refroidissement faibles $(\leqslant 0,5 \mathrm{~K} / \mathrm{min})$ jusqu'à sa température de transition vitreuse. Il est ainsi possible d'effectuer des mesures isothermes sur une plage de plus de $60 \mathrm{~K}$ au-dessous de $T_{\mathrm{f}}$. Des mesures diélectriques ont permis de s'assurer de l'état d'équilibre du produit, et d'étudier la cinétique de cristallisation, spontanée ou provoquée par un traitement thermique cyclique [100].

Il n'existait pas, à notre connaissance, de données relatives à la viscosité du liquide sous-refroidi, mais seulement des résultats obtenus à $T>T_{\mathrm{f}}$ dans un domaine où la viscosité est faible $: \log \eta \lesssim-2$ $[96,98,101]$. Celles que nous présentons ont été obtenues entre $T_{1}=343 \mathrm{~K}$ et $T_{2}=157 \mathrm{~K}$ sur un liquide en équilibre thermodynamique, stable ou métastable, et dans ce cas sans qu'aucune cristallisation ne soit décelable. Les 3 colonnes du tableau I correspondent aux 3 techniques utilisées : écoulement capillaire, rotation, pénétrométrie. On peut remarquer l'accroissement considérable de $\eta$ entre 233 et $157 \mathrm{~K}$ où le rapport des viscosités atteint $10^{8}$, tandis qu'il n'est que de 4 sur un intervalle d'amplitude comparable entre 343 et $273 \mathrm{~K}$.

Le carbonate de propylène utilisé était un produit pour synthèse commercialisé par Merck : pureté $99 \%$, impuretés eau et propylène glycol (analyse par chromatographie en phase gazeuse).

\section{Ajustement de la viscosité selon la relation de Vogel- Fulcher.}

5.1 AJUSTEMENT SUR UN DOMAINe. ETUDE DE LA STABILITÉ DES PARAMÈTRES. - La figure 6 représente les variations de $\bar{T}_{0}, \bar{\delta}_{\mathrm{H}}, \bar{\delta}_{\mathrm{P}}$ observées en éliminant progressivement les valeurs les plus élevées de la viscosité (partie gauche de la Fig. 6) et les valeurs les

Tableau I. - Viscosité du carbonate de propylène $(1 \mathrm{Pl}=1 \mathrm{~Pa} . \mathrm{s})$.

[Viscosity of propylene carbonate.]

\begin{tabular}{cc}
\hline$T / \mathrm{K}$ & $\eta / \mathrm{Pl}$ \\
\hline 343,15 & $1,039 \times 10^{-3}$ \\
343,02 & $1,178 \times 10^{-3}$ \\
333,87 & $1,328 \times 10^{-3}$ \\
331,65 & $1,232 \times 10^{-3}$ \\
324,31 & $1,537 \times 10^{-3}$ \\
315,27 & $1,783 \times 10^{-3}$ \\
312,65 & $2,018 \times 10^{-3}$ \\
306,44 & $2,093 \times 10^{-3}$ \\
296,55 & $2,578 \times 10^{-3}$ \\
294,35 & $2,700 \times 10^{-3}$ \\
288,15 & $2,877 \times 10^{-3}$ \\
276,65 & $3,905 \times 10^{-3}$ \\
&
\end{tabular}

\begin{tabular}{cl}
\hline$T / \mathrm{K}$ & \multicolumn{1}{c}{$\eta / \mathrm{Pl}$} \\
\hline 232,38 & $2,34 \times 10^{-2}$ \\
222,26 & $5,68 \times 10^{-2}$ \\
212,85 & $1,75 \times 10^{-1}$ \\
207,63 & $3,02 \times 10^{-1}$ \\
204,35 & $3,72 \times 10^{-1}$ \\
193,13 & 2,66 \\
& \\
&
\end{tabular}

\begin{tabular}{cc}
\hline$T / \mathrm{K}$ & $\eta / \mathrm{Pl}$ \\
\hline 174,85 & $5,91 \times 10^{3}$ \\
170,75 & $6,66 \times 10^{4}$ \\
167,85 & $3,88 \times 10^{5}$ \\
165,15 & $2,84 \times 10^{6}$ \\
162,65 & $3,20 \times 10^{7}$ \\
162,45 & $5,17 \times 10^{7}$ \\
161,65 & $6,83 \times 10^{7}$ \\
161,25 & $1,08 \times 10^{8}$ \\
160,95 & $1,52 \times 10^{8}$ \\
160,55 & $2,50 \times 10^{8}$ \\
160,25 & $3,16 \times 10^{8}$ \\
158,75 & $1,78 \times 10^{9}$ \\
157,15 & $1,23 \times 10^{10}$ \\
\hline
\end{tabular}




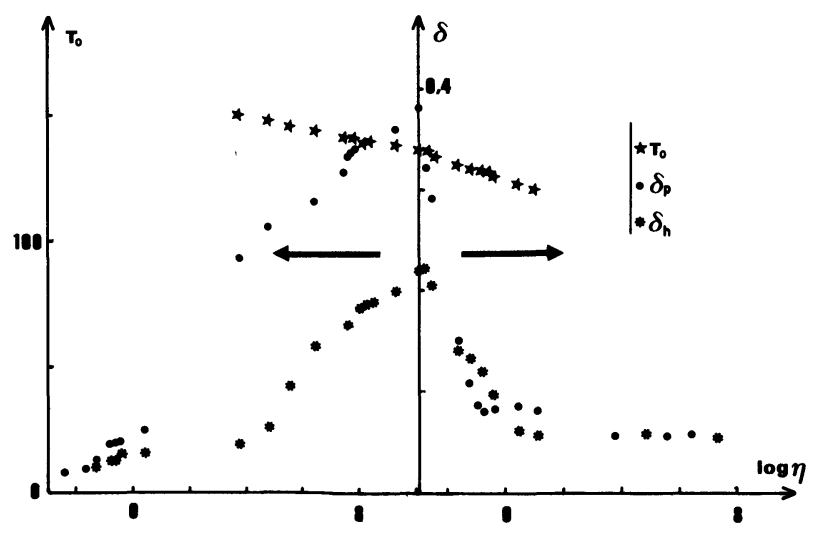

Fig. 6. - Variations de $\bar{T}_{0}, \bar{\delta}_{\mathrm{H}}, \bar{\delta}_{\mathrm{p}}$ observées dans le carbonate de propylène, en fonction de l'intervalle $\left(\log \eta_{1}, \leftarrow\right)$ et $\left(\rightarrow, \log \eta_{2}\right)$.

[Dependence of $\bar{T}_{0}, \bar{\delta}_{\mathrm{H}}, \bar{\delta}_{\mathrm{P}}$ in propylene carbonate to the intervals $\left(\log \eta_{1}, \leftarrow\right)$ and $\left(\rightarrow, \log \eta_{2}\right)$.]
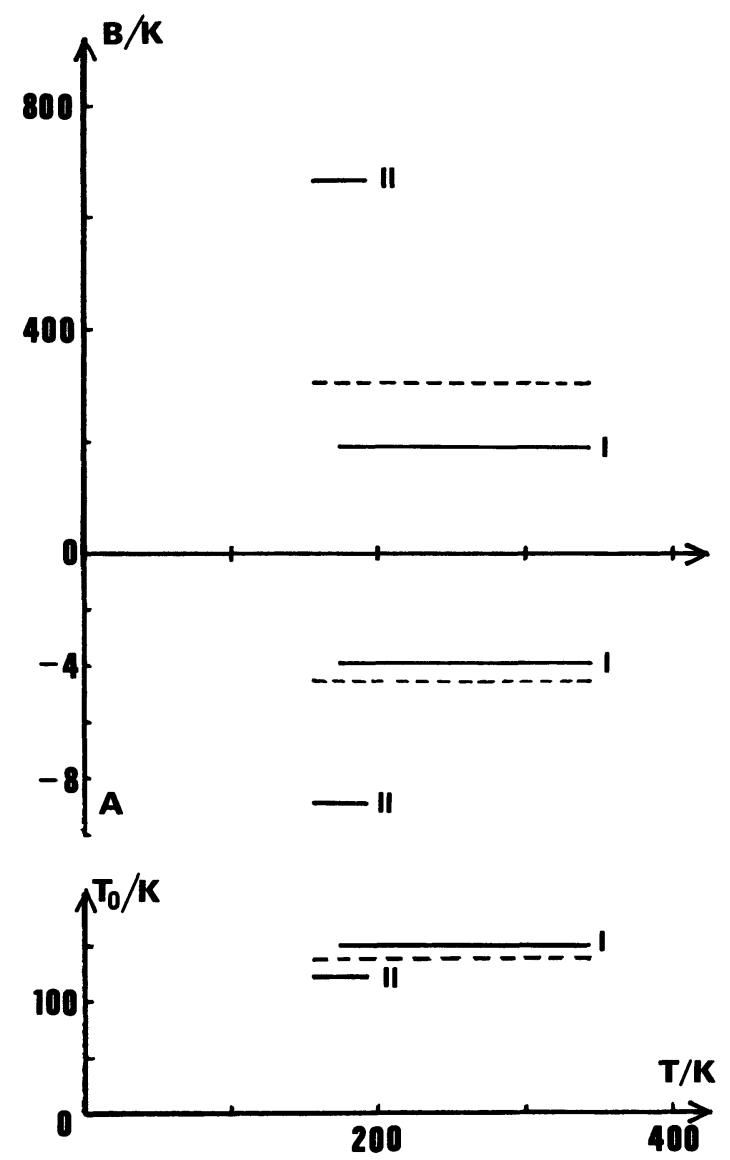

Fig. 7. - Dédoublement des coefficients VFTH consécutif à la séparation de l'intervalle total $\left(T_{1}, T_{2}\right)$ en intervalles partiels I et II. En pointillé, valeur des coefficients de l'ajustement effectué sur $\left(T_{1}, T_{2}\right)$.

[Splitting of the VFTH parameters observed when the whole interval $\left(T_{1}, T_{2}\right)$ is partitioned into 2 partial intervals I and II. Dotted lines : coefficient values fitted on $\left(T_{1}, T_{2}\right)$.] plus faibles (partie droite). Ses caractéristiques diffèrent profondément de celles de la figure $3: \delta_{\mathrm{P}}$ et $\delta_{\mathrm{H}}$ sont dissymétriques et passent toutes deux par un maximum. $T_{0}$ n'est pas stable et présente des variations sensiblement régulières (excepté aux extrémités du diagramme où les ajustements doivent être rejetés après analyse du profil de $\left.\delta_{\mathrm{H}}\left(T_{0}\right)\right)$. Il en est de même de $A$ et $B$. Les ajustements effectués dans les intervalles $\left(T_{1}{ }^{*} \leftarrow\right)$ et $\left(\rightarrow T_{2}{ }^{*}\right)$ avec des écarts types supérieurs à 0,1 environ donnent des distributions de résidus non aléatoires, et sont incompatibles avec la précision expérimentale. Dans ce cas les valeurs correspondantes des paramètres d'ajustement doivent être considérées seulement comme des valeurs moyennes $\bar{A}, \bar{B}, \bar{T}_{0}$ qui sont de simples fonctionnelles de ces intervalles. Il en est ainsi des ajustements $(\mathrm{P})$ et $(\mathrm{H})$ effectués sur l'intervalle total $\left(T_{1}{ }^{*}, T_{2}^{*}\right)$ où les écarts types sont considérables $\left(\bar{\delta}_{\mathrm{P}}=0,38, \bar{\delta}_{\mathrm{H}}=0,22\right)$. Ce résultat est général et recouvre tous les composés que nous avons étudiés. L'ajustement $(\mathrm{H})$ des données sur un intervalle étendu, $\Delta \log \eta \simeq 12$ à 15 , n'est pas valable.

5.2 AJustement en DeUX DOMAINES. - L'ajustement (H) sur l'intervalle complet étant à rejeter, nous avons procédé à des ajustements systématiques sur deux domaines I et II définis par $\log \eta \lesssim 4$ (températures supérieures au "point d'écoulement ") [45] et $\log \eta \gtrsim 2$ (températures inférieures au "point de fusion "). Cette division, très proche d'une partition en deux intervalles, est intéressante car, bien souvent, les données d'origine bibliographique, se trouvent pour des raisons techniques, localisées à l'intérieur de l'un ou l'autre des ces domaines.

Le dédoublement des coefficients engendré par ces deux ajustements partiels est représenté figure 7. Les valeurs des coefficients et des écart types sont données dans le tableau II. On constate que

$$
\begin{aligned}
& \bar{A}_{\mathrm{II}}<\bar{A}<\bar{A}_{\mathrm{I}} \\
& \bar{B}_{\mathrm{I}}<\bar{B}<\bar{B}_{\mathrm{II}} \\
& \bar{T}_{\text {OII }}<\bar{T}_{\mathrm{O}}<\bar{T}_{\text {OI }} .
\end{aligned}
$$

Les deux domaines ne jouent pas un rôle analogue. Les ajustements $(\mathrm{P})$ et $(\mathrm{H})$ apparaissent, selon les composés étudiés, souvent équivalents dans II, tandis que $(\mathrm{H})$ s'avère en général meilleur que $(\mathrm{P})$ dans $\mathrm{I}$. Il en est ainsi pour le carbonate de propylène. D'une façon générale le découpage d'un intervalle en 2 conduit à des ajustements dont les coefficients suivent (6). Ces dédoublements successifs s'interprètent aisément si l'on admet que les paramètres de (1) sont des fonctions de la température $A(T), B(T)$, $T_{0}(T)$ dont on peut obtenir expérimentalement, et selon l'étendue de l'intervalle de mesure, soit une valeur moyenne seulement, soit une valeur " quasi locale $"$.

5.3 Ajustement en $n$ DOMAINEs. - Pour approcher les valeurs locales des paramètres on est donc conduit à découper l'intervalle total $\left(T_{1} T_{2}\right)$ en $n$ intervalles 
Tableau II. - Ajustement de la viscosité du carbonate de propylène suivant la relation de Vogel-Fulcher (1). [Fitting of the propylene carbonate viscosity to the Vogel-Fulcher relation (1).]

\begin{tabular}{cccccccc}
\hline Intervalle de température & $T_{1} / \mathrm{K}$ & $T_{2} / \mathrm{K}$ & $\bar{A}$ & $\bar{B}$ & $\bar{T}_{\mathbf{0}}$ & $10^{2} \bar{\delta}_{\mathbf{P}}$ & $10^{2} \bar{\delta}_{\mathbf{H}}$ \\
\hline Total & 343 & 157 & $-4,55$ & 305 & 137 & 38 & 22 \\
I & 343 & 175 & $-3,91$ & 191 & 150 & 24 & 4,6 \\
II & 193 & 157 & $-8,92$ & 667 & 122 & 8,6 & 6 \\
\hline
\end{tabular}

partiels, dont en principe l'amplitude doit être la plus faible possible. En pratique il faut tenir compte de la nécessité de conserver une amplitude minimum pour disposer d'un nombre suffisant de données à traiter, et obtenir un ajustement $(\mathrm{H})$ significatif, qui soit au moins du $2^{\mathrm{e}}$ ordre. La densité des données concernant le carbonate de propylène nous a permis de procéder à des ajustements sur 6 intervalles d'amplitude $\Delta \log \eta \simeq 7$ se recouvrant partiellement et contenant chacun de 6 à 19 données.

Les variations des coefficients $A_{i}, B_{i}, T_{0 i}(1 \leqslant i \leqslant$ 6), calculés par ce procédé sont représentées figure 8 .
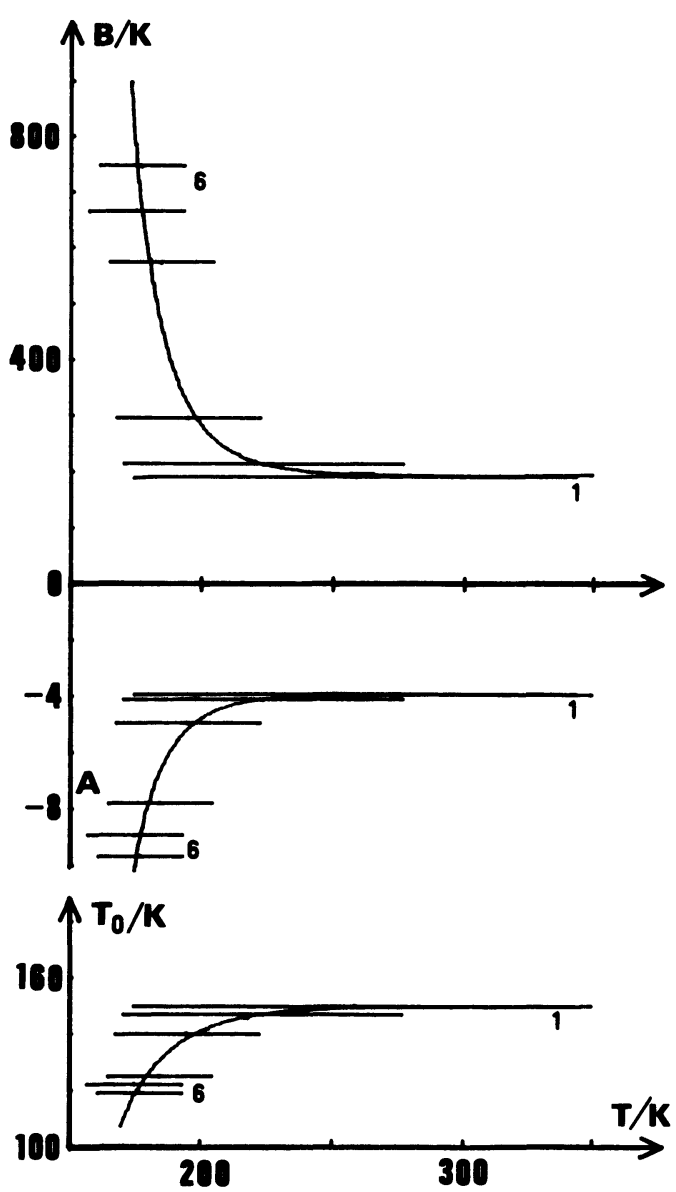

Fig. 8. - Valeurs discrètes des coefficients $A_{i}, B_{i}, T_{0 i}$ (6 intervalles partiels). Les courbes " moyennes " ont été tracées en utilisant (7) et tableau IV.

[Discrete values of $A_{i}, B_{i}, T_{0 i}$ (6 partial intervals). The " mean " curves have been drawn by using (7) and table IV.]
5.3.1 Corrélation des paramètres. - Les variations des coefficients sont fortement corrélées, comme l'indique la figure 9 où sont représentés $A_{i}\left(T_{0 i}\right)$, $B_{i}\left(T_{0 i}\right)$ et $A_{i}\left(B_{i}\right)$. La disposition très régulière des points obtenus après élimination de $T$ permet de les ajuster avec une bonne précision suivant une régression parabolique $A\left(T_{0}\right), B\left(T_{0}\right), A(B)$ (Tableau III).

5.3.2 Variations exponentielles des coefficients VFTH. - L'ajustement en $n$ domaines permet d'approcher les fonctions $A(T), B(T), T_{0}(T)$ par des fonctions constantes par intervalles. Les " courbes moyennes » passant par le milieu de chaque segment (Fig. 8) ont des expressions analytiques de type exponentiel :

$$
y(T)=y_{1} \pm \exp P_{y}(T) .
$$

Les valeurs des coefficients de $P_{y}$ calculées lorsque $P_{y}$ est un polynôme du $2^{\mathrm{e}}$ degré sont indiquées tableau IV.

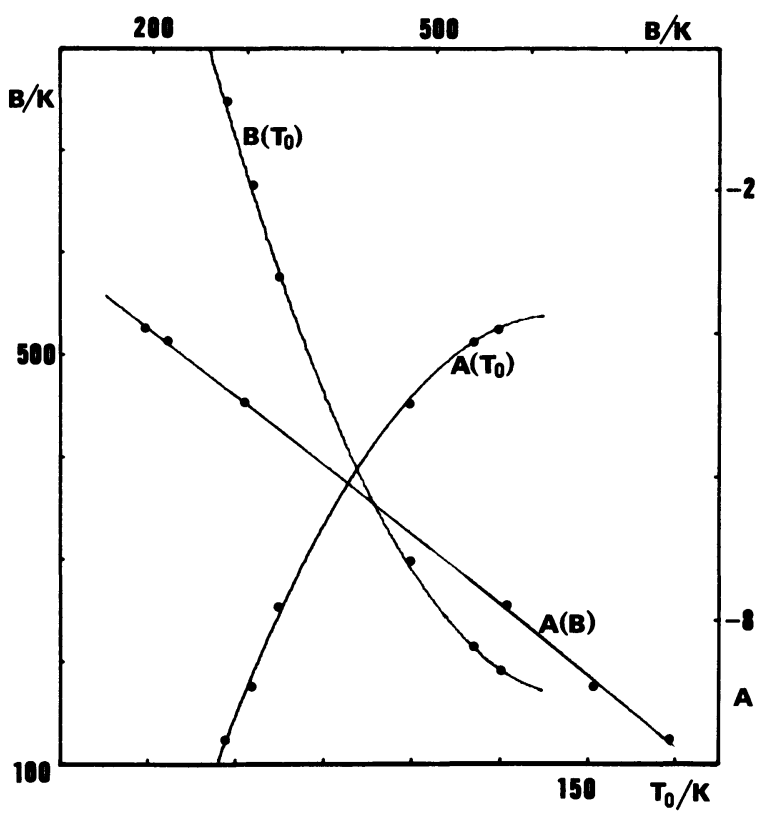

Fig. 9. - Corrélation des coefficients VFTH (6 intervalles partiels). Les courbes ont été tracées avec les coefficients du tableau III.

[VFTH parameters correlation. The curves have been drawn with table III values.] 
Tableau III. - Ajustement des coefficients VFTH locaux suivant une régression polynomiale $y(z)=a z^{2}+b z+c$. [Local VFTH parameters fitted by polynomial regression $y(z)$.]

\begin{tabular}{cccccc}
\hline$y(z)$ & $z$ & $a$ & $b$ & $c$ & $10^{2} \frac{\delta}{|\bar{y}|}$ \\
\hline$A$ & $B$ & $-9,25 \times 10^{-7}$ & $-9,60 \times 10^{3}$ & $-2,02$ & 0,7 \\
$A$ & $T_{0}$ & $-4,27 \times 10^{-3}$ & 1,33 & $-1,08 \times 10^{2}$ & 1,3 \\
$B$ & $T_{0}$ & $3,76 \times 10^{-1}$ & $-1,19 \times 10^{2}$ & $9,58 \times 10^{3}$ & 1,1 \\
\hline
\end{tabular}

Tableau IV. - Ajustement des coefficients VFTH locaux en fonction de la température suivant une régression exponentielle : $y(T)=y_{1} \pm \exp P_{y}(T), P_{y}(T)=a T^{2}+b T+c$.

[Local VFTH parameters fitted against the temperature with an exponential regression.]

\begin{tabular}{lcccc}
\hline$y(T)$ & $a$ & $b$ & $c$ & $10^{2} \delta$ \\
\hline$A(T)$ & $2,36 \times 10^{-4}$ & $-1,66 \times 10^{-1}$ & $2,37 \times 10^{1}$ & 8,7 \\
$B(T)$ & $3,03 \times 10^{-4}$ & $-1,87 \times 10^{-1}$ & $2,99 \times 10^{1}$ & 8,9 \\
$T_{0}(T)$ & $5,47 \times 10^{-5}$ & $-7,12 \times 10^{-2}$ & $1,43 \times 10^{1}$ & 6,3 \\
\hline
\end{tabular}

La relation (7) met en évidence le caractère nettement exponentiel des variations des coefficients VFTH qui tendent asymptotiquement vers une limite sensiblement constante aux températures élevées (Fig. 8). Signalons à ce sujet que des variations de faible amplitude ont été détectées dans cette zone et que le palier haute température pourrait en réalité masquer un extrémum très aplati des coefficients (maximum de $A$ et $T_{0}$, minimum de $B$ ). Des mesures de viscosité effectuées jusqu'à la température d'ébullition $\left(T_{\mathrm{eb}}=513 \mathrm{~K}\right)$ pourraient apporter les informations indispensables pour préciser ce point. On peut aussi noter que la valeur commune $T_{01}=T_{01}=$ $150 \mathrm{~K}$ est identique à celle proposée par Payne et Theodorou [98] en ajustant la viscosité du liquide non surfondu entre $10^{-3}$ et $10^{-1} \mathrm{Pl}$.

Le comportement décrit par (7) ne permet pas de confirmer l'hypothèse selon laquelle $\log \eta$ posséderait un pôle simple à basse température. S'il en était ainsi les courbes $A(T), B(T), T_{0}(T)$ devraient présenter un palier lorsque la température décroît et que $T-T_{0}$ diminue. Ce n'est pas ce qui est observé puisque le palier apparaît à température croissante et que $T_{0 i}$ reste localisé systématiquement quelques dizaines de degrés en dessous de $T_{2 i}$.

Doit-on alors admettre que (1) a son domaine de validité limité aux hautes températures seulement $(T \gtrsim 175 \mathrm{~K})$ et attribuer à $T_{01}$ la signification d'une température de transition thermodynamique caractéristique du liquide? Cela semble difficile, puisque le changement de régime perceptible dès que $T \lesssim 175 \mathrm{~K}$ devrait alors s'expliquer par l'apparition d'un mécanisme physique facilitant l'écoulement du liquide. Différentes hypothèses pourraient être envisagées : effet d'échelle de temps, transition, transformation, cristallisation du carbonate de propylène. Aucune n'est satisfaisante. Un effet d'échelle de temps dû à la proximité de la zone de transition vitreuse $\left(T_{\mathrm{g}}=154 \mathrm{~K}\right.$ d'après nos mesures, $T_{\mathrm{g}}=160 \mathrm{~K}$ d'après celles de Johari et Goldstein [102] en ATD) est en effet à écarter car le liquide a été étudié dans un état d'équilibre entre $175 \mathrm{~K}$ (temps de relaxation diélectrique $\left.\tau_{0} \simeq 10^{-6} \mathrm{~s}\right)$ et $157 \mathrm{~K}\left(\tau_{0} \simeq 10 \mathrm{~s}\right)$ et ne présente alors aucun phénomène d'hystérésis. Un processus de cristallisation, même trop faible pour être décelable par spectroscopie diélectrique ou analyse de $h^{2}(t)$ ne saurait expliquer le changement de régime d'écoulement, puisqu'il conduirait à une augmentation de $K(3)$, et à une diminution de $\eta$, alors que H(I) surestime déjà les valeurs mesurées dans II. Enfin ni les mesures de permittivité complexe et de conductivité électrique [100] ni des mesures thermodynamiques n'ont permis de détecter la moindre anomalie signalant une transition ou transformation du liquide.

Tout cela nous conduit à ne pas privilégier la zone (I) et à conclure que la relation VFTH est une relation locale seulement, en général équivalente à une approximation du $2^{\mathrm{e}}$ ordre, et qui peut être utilisée sous la forme généralisée

$$
\log \eta=A(T)+\frac{B(T)}{T-T_{0}(T)}
$$

Les coefficients VFTH apparaissent donc être des fonctions de la température dont nous proposons une expression analytique approchée à l'aide de la relation (7). 
5.3.3 Précision des ajustements. - L'étude précédente permet d'estimer quelle est l'influence du nombre de paramètres utilisés sur la précision des ajustements de $\log \eta$.

Avec seulement 3 paramètres, l'ajustement ( $\mathrm{H}$ ou $\mathrm{P}$ ) doit être rejeté vu son écart type nettement trop élevé $\left(\bar{\delta}_{\mathrm{H}}=0,22, \bar{\delta}_{\mathrm{P}}=0,38\right)$ tandis qu'avec 6 paramètres $\left(\mathrm{H}_{\mathrm{I}}\right.$ et $\left.\mathrm{H}_{\mathrm{II}}\right)$ il apparaît tout à fait compatible avec la précision expérimentale et peut donc être conservé $\left(\delta_{\mathrm{H}}=5,4 \times 10^{-2}\right)$. L'utilisation d'un jeu de fonctions élaborées à l'aide de (7), tableaux III et IV, soit 10 paramètres, n'améliore pas $\delta_{\mathrm{H}}$, bien au contraire, ce qui s'explique par les erreurs supplémentaires $\Delta$ introduites par l'ajustement de ces fonctions :

$$
\Delta \log \eta \simeq \Delta A+0,02 \Delta B+0,4 \Delta T_{0} .
$$

Ces erreurs pourraient d'ailleurs être facilement réduites en affinant la corrélation (5.3.1) et les lissages (7) avec des polynômes de degré plus élevé. L'emploi des 18 paramètres donnés par le découpage en 6 intervalles partiels ne diminue pas non plus $\delta_{\mathrm{H}}\left(\delta_{\mathrm{H}} \simeq 8 \times 10^{-2}\right)$ à cause de la pondération des données induite par le chevauchement des intervalles. Bien que la méthode utilisée optimise le mode de découpage, il subsiste néanmoins une certaine latitude dans le choix des intervalles, ce qui se traduit par des écart types de l'ordre de 5 à $8 \times 10^{-2}$. C'est ainsi qu'un découpage en 8 intervalles (24 paramètres) donne $\delta_{\mathrm{H}} \simeq 7 \times 10^{-2}$. Un ajustement suivant deux nouveaux domaines (I : 343-193 K, II : 175-157 K) permet de réduire $\delta$ à $4,7 \times 10^{-2}$ en utilisant $(\mathrm{H})$ dans I et (P) dans II. Toutes ces variations ne nous semblent pas très significatives, car elles résultent avant tout du mode de traitement des données, et du poids excessif que possède individuellement chaque mesure, vu le nombre total relativement limité de données (31). Elles nous permettent de retenir comme acceptables et sensiblement équivalents tous les ajustements dont la variance est comprise entre environ 3 et $6 \times 10^{-3}$, ce qui est réalisé en utilisant 6 paramètres ou plus, et ne l'est pas du tout avec 3 paramètres (48 et $144 \times 10^{-3}$ pour $\mathrm{H}$ et $\mathrm{P}$ ).

A l'issue de cette étude nous considérons que l'ajustement de la viscosité du carbonate de propylène, à l'aide de deux régressions $\mathrm{H}$ ou d'une régression $\mathrm{H}$ à haute température et $\mathrm{P}$ à basse température, permet d'obtenir des formules d'interpolation valables dans deux domaines distincts, et offre donc un intérêt pratique indéniable, mais ne traduit nullement un changement du régime d'écoulement. La relation généralisée (8), elle, ne présente aucun intérêt pratique comme formule d'interpolation vu les difficultés techniques qu'il faut résoudre pour parvenir à l'établir. Par contre elle montre clairement que la relation de Vogel a un caractère local seulement, et que ses coefficients varient progressivement au cours du refroidissement du liquide.

\section{Interprétation des résultats. Modèles du volume} libre.

Les modèles fondés sur le volume libre conduisent à une expression de la viscosité établie par Doolittle $[4,5]$

$$
\eta=\eta_{\infty} \exp \left(\frac{1}{f}\right)
$$

où $\eta_{\infty}$ est une constante et $f$ la concentration du volume libre. Rappelons que c'est à la suite des difficultés rencontrées pour représenter les variations thermiques de $\eta$ dans une série homologue d'alcanes normaux que Doolittle avait proposé cette relation en déterminant expérimentalement le volume libre par des mesures du volume spécifique et par leur extrapolation à $0 \mathrm{~K}$. Cette relation s'est avérée très générale, voire " universelle " si l'on convient de définir $f(T)$ selon une formulation analytique propre à chaque modèle. La méthode d'approche de $\log \eta$ que nous avons développée possède son équivalent en terme de volume libre et permet d'obtenir une estimation de $f$ définie par (10), indépendante de chaque modèle.

6.1 Modèle ClassiQue du volume LiBRe [8, 47]. Les relations VFTH et Doolittle sont alors identiques et $f(T)$ est proportionnel à $T-T_{0}$ (pour $T \geqslant T_{0}$ ). Le rejet de (1) entraîne celui de (10). Des variations linéaires de $f(T)$ sur tout l'intervalle $\left(T_{1}, T_{2}\right)$ sont inacceptables et le modèle classique est en défaut et doit être écarté.

6.2 Equation De Doolittle GÉNÉRAlisée. - La validité locale de (1) et sa généralisation suivant (8) permettent de définir une concentration du volume libre $f^{(1)}$ à l'aide d'une relation de Doolittle identique à (8) où $\eta_{\infty}$ n'est plus une constante mais une fonction de $T$. La décomposition en intervalles partiels établit la correspondance entre les divers coefficients :

$$
\begin{aligned}
A_{i} & =\log \eta_{\infty i} \\
f_{i}^{(1)}(T) & =\left(T-T_{0 i}\right) \frac{\log e}{B_{i}} .
\end{aligned}
$$

Au point de vue géométrique, l'approche de $\log \eta$ (au $2^{\mathrm{e}}$ ordre) par une famille de $n$ hyperboles est ainsi équivalente à l'approche (au $1^{\mathrm{er}}$ ordre) de $f^{(1)}$ à l'aide de fonctions linéaires $f_{i}^{(1)}$ dont elle constitue l'enveloppe (Fig. 10)).

6.3 Modèle de Cohen et Grest $[59,61]$. - Le modèle proposé par Cohen et Grest substitue à la relation à 3 paramètres VFTH une relation à 4 paramètres

$\log \eta=\alpha+\frac{2 \beta}{T-T_{\infty}+\left(\left(T-T_{\infty}\right)^{2}+\gamma T\right)^{1 / 2}}$ 


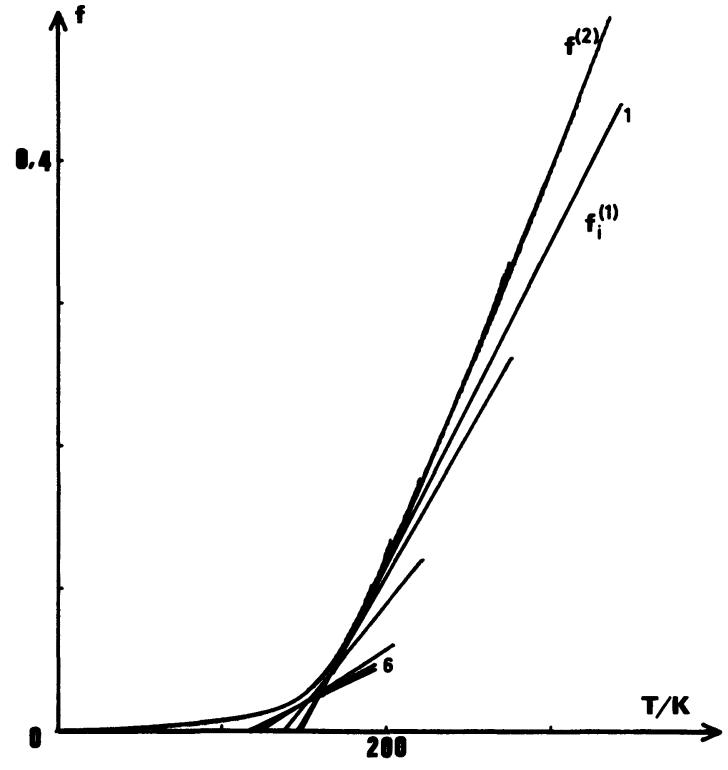

Fig. 10. - Variations thermiques de la concentration du volume libre défini par l'équation de Doolittle : $f_{i}^{(1)}$ approche linéaire locale avec $\eta_{\infty}(T)=10^{A(T)}, f^{(2)}$ modèle de Cohen et Grest.

[Temperature dependence of the Doolittle free-volume fraction $f_{i}^{(1)}$ local linear approximation with $\eta_{\infty}(T)=$ $10^{A(T)}, f^{(2)}$ Cohen-Grest model.]

La concentration correspondante de volume libre $f^{(2)}$ n'est plus linéaire comme dans le modèle classique, mais dépend de 3 paramètres dont l'un, $\gamma$, peut être considéré comme un facteur correctif, qui lorsqu'il est nul réduit (13) à (1)

$f^{(2)}(T)=\left(T-T_{\infty}+\left(\left(T-T_{\infty}\right)^{2}+\gamma T\right)^{1 / 2}\right) \frac{\log e}{2 \beta}$.

Les données du CP (Tableau I) ont été traitées suivant une régression linéaire à 2 paramètres $\alpha$ et $\beta$, en utilisant $\gamma$ et $T_{\infty}$ comme variables auxiliaires. Les valeurs des coefficients sont données tableau V et l'ajustement est considéré comme satisfaisant, compte tenu de la faible valeur de sa variance $\delta^{2} \simeq 5 \times 10^{-3}$. La courbe $f^{(2)}(T)$ varie continûment et ne s'annule qu'à $0 \mathrm{~K}$, elle est, bien que distincte, très proche de l'enveloppe des $f_{i}^{(1)}(T)$ (Fig. 10).

Tableau V. - Ajustement de la viscosité du carbonate de propylène suivant la relation de Cohen-Grest (13).

[Fitting of the propylene carbonate viscosity to the Cohen-Grest relation (13).]

\begin{tabular}{ccccccc}
$T_{1} / \mathrm{K}$ & $T_{2} / \mathrm{K}$ & $\alpha$ & $\beta$ & $\gamma$ & $T_{\infty}$ & $10^{2} \delta$ \\
\hline 343 & 157 & $-3,76$ & 152 & 5,7 & 166 & 7,2
\end{tabular}

La courbe $T_{0}^{(1)}$ définie par

$$
T_{0}^{(1)}(T)=T-\frac{f^{(2)}}{f^{\prime(2)}}
$$

présente des variations analogues à celles de $T_{0 i}$, bien qu'il apparaisse un décalage systématique qui résulte des définitions différentes de $f^{(1)}$ et $f^{(2)}$ (Fig. 11).

Ce décalage disparaît si l'on identifie (10) et (1) sur chaque intervalle partiel, ce qui conduit alors à une approche locale plus rigoureuse de $f$ au moyen de fonctions $f_{i}^{(2)}$ sensiblement quadratiques en $T$ :

$$
\begin{aligned}
f_{i}^{(2)} & =f_{i}^{(1)} \frac{1}{1+k_{i}} \\
k_{i} & =\frac{A_{i}-\log \eta_{\infty}}{B_{i}}\left(T-T_{0 i}\right) .
\end{aligned}
$$

Les relations $(12,16,17)$ qui sont totalement indépendantes de la relation de Cohen-Grest (14) permettent de construire à l'aide de $f_{i}^{(2)}$, la fonction $f$ définie par la relation de Doolittle (10). Le résultat obtenu montre que $f$ et $f^{(2)}$ sont pratiquement identiques (Fig. 10). La concentration de volume libre estimée selon notre méthode coïncide donc avec celle du modèle de Cohen-Grest. Les relations (13) et (8), sont équivalentes et permettent de définir $T_{0}^{(2)}(T)$ par

$$
\begin{aligned}
T_{0}^{(2)}(T) & =T-\frac{f^{(2)}}{f^{(2)}} \cdot \frac{1}{1+k} \\
\frac{1}{1+k} & =1-\frac{A(T)-\log \eta_{\infty}}{\log e} f^{(2)} .
\end{aligned}
$$

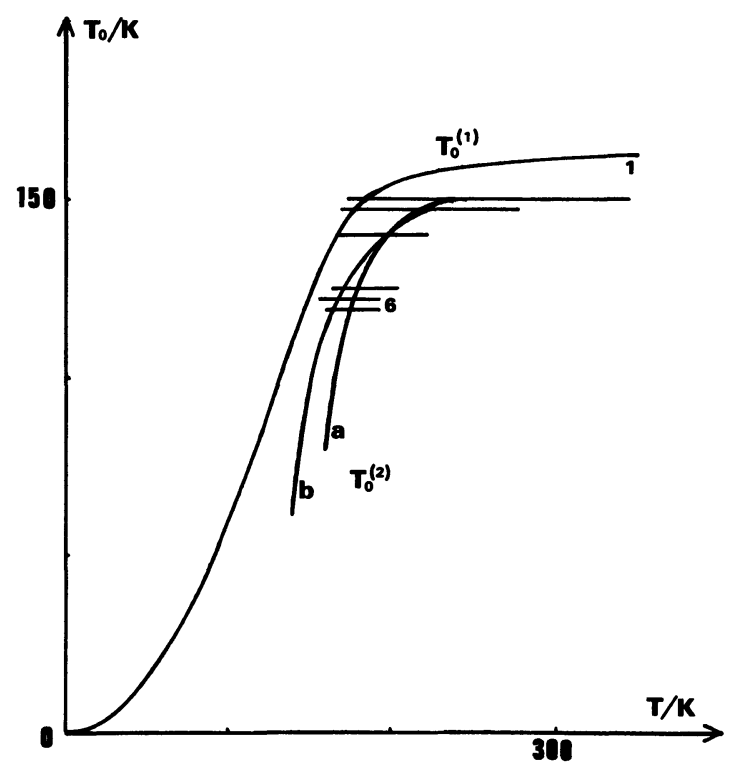

Fig. 11. - Variations de $T_{0}(T)$ défini par (15) et (18) avec : (a) les valeurs du tableau $\mathrm{V}$, (b) les valeurs $T_{\infty}=150$, $\alpha=-4,24, \beta=473, \gamma=3,8$.

[Temperature dependence of $T_{0}(T)$ following (15) and (18) with : (a) the table $\mathrm{V}$ values, (b) the values $T_{\infty}=150$, $\alpha=-4.24, \beta=473, \gamma=3.8$.] 
L'accord entre les variations de $T_{0}^{(2)}(T)$ et de $T_{0 i}$ (Fig. 11) apparaît tout à fait acceptable.

On voit ainsi qu'il y a équivalence entre la relation de Cohen et Grest et la relation de Vogel-Fulcher généralisée. Toutes deux conduisent à la même estimation du volume libre.

\section{Conclusion.}

L'étude de la viscosité du carbonate de propylène fait ressortir le rôle essentiel joué par les fenêtres d'observation et de mesure $\Delta T, \Delta T^{*}, \Delta \log \eta$ et montre l'intérêt de disposer de résultats couvrant toute la gamme liquide s'étendant depuis la température d'ébullition jusqu'à la température de transition vitreuse. A cette échelle, il apparaît clairement que ni la relation d'Arrhenius, ni celle de VogelFulcher ne sont valables et que le modèle classique de Cohen et Turnbull doit être abandonné. Notre analyse ne nous a pas permis de mettre en évidence l'existence d'une température caractéristique du liquide qui serait un pôle simple de $\log \eta$. Elle a, par contre, révélé que les coefficients VFTH ne sont pas des constantes mais des fonctions $A(T), B(T), T_{0}(T)$ de la température. Ce résultat nous permet d'avancer l'hypothèse selon laquelle il existe un spectre continu de paramètres VFTH. Dans ce cas les valeurs discrètes $\bar{A}, \bar{B}, \bar{T}_{0}$ calculées par ajustement peuvent représenter, suivant l'étendue et la localisation de $\Delta T^{*}$, des valeurs moyennes ou locales des fonctions $A(T)$, $B(T), T_{0}(T)$. C'est ce que nous avons établi non seulement dans le carbonate de propylène, mais aussi dans le glycérol et dans d'autres composés [103].

L'hypothèse d'un spectre continu de coefficients explique alors toutes les difficultés rencontrées habituellement sur le plan expérimental pour ajuster les données suivant (1) ou la relation WLF, et pour localiser correctement $T_{0}(\S 1)$. L'emploi d'un spectre discret de plusieurs triplets garde évidemment un intérêt pratique incontestable, et permet d'obtenir des formules d'interpolation excellentes, mais dont l'extrapolation est totalement injustifiée et conduit toujours à plus basse température à une surestimation de la viscosité.

Les variations de la viscosité du carbonate de propylène peuvent aussi être analysées à l'aide du volume libre relatif $f$ défini opérationnellement par l'équation de Doolittle. Deux concentrations $f^{(1)}$ et $f^{(2)}$ sont à considérer selon que l'on exprime le facteur préexponentiel à l'aide de $A(T)$ ou qu'on lui impose de rester constant. Les deux fonctions sont proches l'une de l'autre et présentent une courbure analogue; de plus la concentration $f^{(2)}$ se révèle être identique à celle du modèle de Cohen et Grest. L'hypothèse d'un spectre continu de coefficients VFTH a une interprétation très claire en termes de volume libre puisqu'elle traduit simplement des variations non linéaires de $f(T)$. Le modèle de Cohen et Grest est en accord avec cette hypothèse et présente le grand intérêt d'offrir un ajustement de $\log \eta$ à 4 paramètres avec une précision voisine de celle obtenue avec 6 paramètres (deux triplets $\mathrm{H}$ ou $\mathrm{P}$ ). Il procure ainsi une relation d'interpolation très satisfaisante sur une quinzaine de décades. Ce résultat n'est certes pas suffisant pour juger de la validité du modèle qui sur le plan théorique interprète la vitrification par un processus de percolation. Il est fondé sur l'existence d'une structure en cages avec des cellules " quasi-liquides » (qui seules ont un volume libre) regroupées en amas, dans lesquels la diffusion moléculaire n'est possible que si leur taille est suffisante. La concentration de cellules " quasi-liquides » varie suivant la température et passe par une valeur critique conduisant 'à la formation d'un amas infini. D'autres mesures, thermodynamiques (dilatation, chaleur spécifique), diélectriques, à pression variable seraient indispensables pour accéder aux paramètres moléculaires du modèle, ce que ne permet pas la seule étude de la viscosité isobare.

La méthode que nous présentons, permet d'estimer si un modèle est compatible avec les données expérimentales recueillies sur une gamme très étendue. Dans le cas du carbonate de propylène, il en est bien ainsi pour le modèle de Cohen-Grest, tout au moins en ce qui concerne la viscosité.

\section{Bibliographie}

[1] VoGel, H., Phys. Z. 22 (1921) 645-6.

[2] Fulcher, G. S., J. Amer. Ceram. Soc. 8 (1925) 339-55.

[3] Tammann, G., Hesse, W., Z. Anorg. Allgem. Chem. 156 (1926) 245-57.

[4] Doolittle, A. K., in Alexander Ed., Colloid Chemistry (Reinhold) 1950, vol. 7, 149-73.

[5] Doolittle, A. K., J. Appl. Phys. 23 (1952) 236-9.

[6] Davidson, D. W., Cole, R. H., J. Chem. Phys. 19 (1951) 1484-90.

[7] Dienes, G. J., J. Appl. Phys. 24 (1953) 779-82.

[8] Cohen, M. H., Turnbull, D., J. Chem. Phys. 31 (1959) 1164-69.
[9] Barlow, A. J., Lamb, J., Matheson, A. J., Proc. $R$. Soc. London A 292 (1966) 322-42.

[10] Davies, D. B., Matheson, A. J., J. Chem. Phys. 45 (1966) 1000-6.

[11] Greet, R. J., Turnbull, D., J. Chem. Phys. 46 (1967) 1243-51.

[12] Barlow, A. J., Lamb, J., Dis. Farad. Soc. 43 (1967) 223-30.

[13] Carpentier, M. R., Davies, D. B., Matheson, A. J., J. Chem. Phys. 46 (1967) 2451-54.

[14] Berberian, J. G., Cole, R. H., J. Amer. Chem. Soc. 90 (1968) 3100-4. 
[15] McCall, D. W., Douglas, D. C., Falcone, D. R., J. Chem. Phys. 50 no 9 (1969) 3839-43.

[16] Chang, S. S., Bestul, A. B., J. Chem. Phys. 56 (1972) 503-16.

[17] Laughlin, W. T., Uhlmann, D. R., J. Phys. Chem. 76 (1972) 2317-25.

[18] HARRISON, G., The dynamic properties of supercooled liquids (Academic Press London) 1976.

[19] Calemczuk, R., Thèse, Grenoble 1977.

[20] MAZIÈRes, C., Les solides non cristallins (P.U.F. Paris) 1978.

[21] Wilson, W. L., Mellon, P. F., Berberian, J. G., J. Chem. Phys. 76 no 5 (1982) 2602-5.

[22] Angell, C. A., Torell, L. M., J. Chem. Phys. 78 (1983) $937-45$

[23] Berberian, J. G., J. Chem. Phys. 79 (1983) 5077-9.

[24] Robinson, H. A., Peterson, C. A., J. Amer. Ceram. Soc. 27 (1944) 129-138.

[25] Hagy, H. E., J. Amer. Ceram. Soc. 46 (1963) 93-7.

[26] Napolitano, A., Hawkins, E., J. Res. Natl. Bur. Stand. (USA) 68A (1964) 439-48.

[27] Napolitano, A., Macedo, P. B., Hawkins, E. G., J. Amer. Ceram. Soc. 48 (1965) 613-6.

[28] Napolitano, A., Hawkins, E., N.B.S. Misc. Pub. 260 (1966) 1-24.

[29] Macedo, P. B., Litovitz, T. A., J. Chem. Phys. 42 (1965) 245-56.

[30] Hu, H., Mackenzie, J. D., J. Non Cryst. Solids 54 $\mathrm{n}^{\circ} 3$ (1983) 241-51.

[31] Williams, M. L., Landel, R. F., Ferry, J. D. J. Amer. Chem. Soc. 77 (1955) 3701-7.

[32] McGrum, N. G., Read, B. G., Williams, G., Anelastic and dielectric effects in polymeric solids (Wiley London) 1967.

[33] ChampetieR, G., Chimie macromoléculaire (Hermann Paris) 1970.

[34] Voir par exemple Images de la Physique, Supp. au

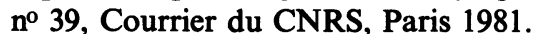

[35] Chen, H. S., Goldstein, M., J. Appl. Phys. 43 (1972) 1642-8.

[36] Anderson, P. M., Lord, A. E., J. Non Cryst. Solids 37 (1980) 219-29.

[37] Johari, G. P., Philos. Mag. B 46 (1982) 549-64.

[38] Johari, G. P., Goodby, J. W., J. Chem. Phys. 77 $\mathrm{n}^{\circ} 10$ (1982) 5165-72.

[39] Schad, H., Zeller, H. R., Phys. Rev. A 26 no 5 (1982) 2940-5.

[40] Zeller, H. R., Phys. Rev. A 26 no 3 (1982) 1785-7.

[41] AmoureuX, J. P., Foulon, M., Bee, M., Noyel, G., Jorat, L., Mol. Phys. 52 (1984) 161-71.

[42] Courtens, E., Phys. Rev. Lett. 52 (1984) 69-72.

[43] Angell, C. A., Proc. workshop on Relaxation Processes. Blacksburg, V. A., Ngai Ed. (1983).

[44] Voir par exemple Rapport de prospective DGRST (La Documentation Française, Paris) 1980.

[45] ZARZYCKI, J., Les verres et l'état vitreux (Masson, Paris) 1982.

[46] Dworkin, A., Fuchs, A. H., Ghelfenstein, M., SzWARC, H., J. Phys. Lett. 43 no 1 (1982) L21-7.

[47] Turnbull, D., Cohen, M. H., J. Chem. Phys. 34 (1961) 120-5.

[48] Gibbs, J. H., Di Marzio, E. A., J. Chem. Phys. 28 (1958) 373-83.

[49] Adam, G., Gibrs, J. H., J. Chem. Phys. 43 (1965) $139-46$.
[50] Kauzmann, W., Chem. Rev. 48 (1948) 219-56.

[51] GoldSTEIN, M., in Mackenzie, Modern aspects of the vitreous state (Butterworth London) 1964, vol. 3.

[52] Goldstein, M., J. Chem. Phys. 51 no 9 (1969) 3728-39.

[53] Goldstein, M., J. Chem. Phys. 64 (1976) 4747-74.

[54] Goldstein, M., J. Chem. Phys. 67 (1977) 2246-53.

[55] Haddad, J., Goldstein, M., J. Non Cryst. Solids 30 no 1 (1978) 1-22.

[56] ANDERson, P. W., in Balian R. et al. Ed., La matière mal condensée, Les Houches 1978 (North Holland) 1979, p. 159.

[57] Gujrati, P. D., Goldstein, M., J. Phys. Chem. 84 no $^{\circ}$ (1980) 859-63.

[58] Eastwood, A. R., J. Chem. Soc. Farad. Trans. 2 77 (1981) 1411-20.

[59] Cohen, M. H., Grest, G. S., Phys. Rev. B 20 (1979) 1077-98

[60] Grest, G. S., Cohen, M. H., in Adv. in Chemical Phys. Prigogine Ed. (Wiley, New York) 1981, t. 48, p. 455.

[61] Cohen, M. H., Grest, G. S., Phys. Rev. B 26 (1982) 2664-5.

[62] Cohen, M. H., Grest, G. S., J. Non Cryst. Solids 61-62 (1984) 749-60.

[63] JofFRIN, J., in Balian R. et al. Ed. La matière mal condensée, Les Houches 1978 (North Holland) 1979, p. 106-57.

[64] Toulouse, G., in Congrès de la Société Française de Physique. Clermont-Ferrand 1981.

[65] Cyrot, M., J. Phys. C 8 (1980) 107-9.

[66] Cyrot, M., Phys. Lett. A 83 (1981) 275-8.

[67] Duffy, D. M., Rivier, N., J. Physique Colloq. 43 (1982) C9-475-81.

[68] Davies, D. B., Matheson, A. J., Disc. Farad. Soc. 43 (1967) 216-22.

[69] Davies, D. B., Matheson, A. J., Trans. Farad. Soc. 63 (1967) 596-603.

[70] Turnbull, D., Cohen, M. H., J. Chem. Phys. 52 (1970) 3038-41.

[71] Dworkin, A., J. Chim. Phys. 71 (1974) 929-30.

[72] Turnbull, D., Bagley, B. G., in Treatise on solid state chemistry, Hannay N. B. Ed. (Plenum Press) 1975, vol. 5.

[73] Wong, J., ANGell, C. A., Glass structure by spectroscopy (Dekker, New York) 1976.

[74] Kovacs, A. J., J. Polym. Sci. 30 (1958) 131-47.

[75] Sanchez, J. C., J. Appl. Phys. 45 (1974) 4204-15.

[76] Khong, T. D., Rheolog. Acta 17 no 6 (1978) 654-62.

[77] Schwarzl, F. R., in Rheology, Astarita et al. Ed. (Plenum Press) 1980, vol. 1.

[78] Angell, G. A., RaO, K. J., J. Chem. Phys. 57 (1972) 470-81

[79] Tweer, H., J. Chem. Phys. 54 (1971) 1952-9.

[80] Hill, R. M., Dissado, L. A., J. Phys. C 15 (1982) 5171.

[81] Utracki, L. A., J. Macrom. Sci. Phys. B 10 (1974) 477-505.

[82] Utracki, L. A., J. Macrom. Sci. Phys. B 18 (1980) 731-74.

[83] Yagihara, S., Mashimo, S., J. Polym. Sci. Polym. Phys. Ed. 19 no 9 (1981) 1333-37.

[84] Fontana, E. H., Plummer, W. A., J. Amer. Ceram. Soc. 62 (1979) 367-9.

[85] Sturm, K. G., Rheol. Acta 20 (1981) 59-63.

[86] BondeaU, A., Thèse 1985, Lyon. 
[87] Persoz, B., Vourlat, P., Techniques de l'Ingénieur, fasc. p. 3305 .

[88] Bondeau, A., Huck, J., Revue Phys. Appl. 18 (1983) 307-12.

[89] Smith, T. L., Ferry, J. D., Schremp, F. W., J. Appl. Phys. 20 (1949) 144-53.

[90] Bacon, J. F., Hasapis, A. A., Wholley, J. W., Phys. Chem. Glasses 1 (1960) 90-8.

[91] Kurkjian, C. R., Douglas, R. W., Phys. Chem. Glasses 1 (1960) 19-25.

[92] Leko, B. K., Sov. J. Glass Phys. Chem. 5 (1979) 228-47.

[93] Orbon, S. J., J. Polym. Sci. Polym. Phys. 20 no 9 (1982) 1575-83.

[94] JASINSKI, R., in Advances in Electrochemistry and Electrochemical Engineering, Delahay P., Tobias C. W. (Ed.), Vol. 8 (John Wiley and Sons, Inc. New York) 1971, 253-335.

[95] Cavell, E. A., J. Chem. Soc. Faraday Trans. 270 (1974) 78-84.
[96] Casteel, J. F., Angel, J. R., McNeeley, H. B., Sears, P. G., J. Elect. Soc. 122 (1975) 319-24.

[97] Simeral, S. L. and Amey, R. L., J. Chem. Phys. 74, 7 (1970) 1443-1446.

[98] Payne, G. R. and Theodorou, I. E., J. Phys. Chem. 76 (1972) 2892-2900.

[99] Courtot-Coupez, J., Madec, C., C.R. Hebd. Séan. Acad. Sci. C 277 (1973) 15-8.

[100] HuCK, J., Noyel, G. A., Jorat, L. J., Bondeau, A. M., J. Electrostatics 12 (1982) 221-8. Voir aussi 7th Int. Conf. on Conduction and Breakdown in Dielectric Liquids. Berlin 1981. Schmidt Ed.

[101] Masood, A. K., Pethrick, R. A. and Swinton, F. L., J. Chem. Soc. Faraday Trans. 172 (1976) 20-28.

[102] Johari, G. P. and Goldstein, M., J. Chem. Phys. 536 (1970) 2372-2388.

[103] Bondeau, A. M., HuCK, J. R. (à paraître). 